Meta

Journal des traducteurs

Translators' Journal

\title{
Détermination et figement au regard de la traduction
}

\section{Pierre-André Buvet}

Volume 53, numéro 2, juin 2008

La traduction des séquences figées

The Translation of Frozen Sequences

URI : https://id.erudit.org/iderudit/018523ar

DOI : https://doi.org/10.7202/018523ar

Aller au sommaire du numéro

Éditeur(s)

Les Presses de l'Université de Montréal

ISSN

0026-0452 (imprimé)

1492-1421 (numérique)

Découvrir la revue

Citer cet article

Buvet, P.-A. (2008). Détermination et figement au regard de la traduction. Meta, 53(2), 333-364. https://doi.org/10.7202/018523ar

\section{Résumé de l'article}

Les corrélations entre la détermination et le figement sont souvent étudiées au sein de séquences figées de nature non déterminative. Il existe néanmoins de nombreuses constructions déterminatives à caractère figé. L’objectif de cet article est d'en faire l'inventaire et de montrer l'intérêt d'un tel recensement dans la perspective de la traduction automatique. 


\title{
Détermination et figement au regard de la traduction
}

\author{
PIERRE-ANDRÉ BUVET \\ Université Paris 13, Paris, France \\ pab@lli.univ-paris13
}

\begin{abstract}
RÉSUMÉ
Les corrélations entre la détermination et le figement sont souvent étudiées au sein de séquences figées de nature non déterminative. Il existe néanmoins de nombreuses constructions déterminatives à caractère figé. L'objectif de cet article est d'en faire l'inventaire et de montrer l'intérêt d'un tel recensement dans la perspective de la traduction automatique.
\end{abstract}

\begin{abstract}
The correlations between determination and frozen expressions are generally studied within the framework of set phrases of a non-determinative nature. Nonetheless, there are numerous determinative constructions of a frozen nature. Our main objective in this article is to do an inventory of this kind of phrase and to show its value in the context of natural language processing.

\section{MOTS-CLÉS/KEYWORDS}

déterminant, détermination, figement, modifieur, traduction automatique
\end{abstract}

\section{Introduction}

Nous considérons que le développement de systèmes de traduction de qualité (automatique ou semi-automatique) doit être fondé prioritairement sur des descriptions formalisées des données linguistiques. La présente étude est une illustration de ce point de vue. Dans un premier temps, nous indiquons ce que recouvrent les termes déterminant, prédéterminant, modifieur et détermination, puis nous faisons état des différentes formes déterminatives du français afin d'établir par la suite lesquelles sont concernées par le figement. Dans un deuxième temps, nous distinguons trois catégories de phénomènes relatifs conjointement au figement et à la détermination: les déterminants des séquences figées, les modifieurs figés et les déterminants complexes figés; après avoir écarté de notre champ d'études la première catégorie, nous établissons ce qui distingue la deuxième catégorie de la troisième. Dans un troisième temps, nous mettons en évidence diverses particularités sémantiques des modifieurs et déterminants complexes figés du français. Enfin, dans la perspective de la traduction automatique, nous discutons des représentations métalinguistiques standardisées qu'il convient d'associer aux séquences déterminatives étudiées et nous montrons l'intérêt de telles représentations pour des systèmes de traduction. 


\section{La détermination: définition et typologie}

Après avoir défini la détermination et évoqué certaines de ses caractéristiques, nous présentons une classification de la détermination afin d'établir quelles formes déterminatives ressortissent au figement.

\subsection{La notion de déterminant}

Nous mentionnons tout d'abord le cadre théorique de notre étude et la taxinomie des unités linguistiques qui en résulte dans la mesure où cette dernière participe à la définition de la détermination que nous proposons ensuite.

Le modèle des classes d'objets (Gross 1994, 1995a et 1995b; Le Pesant et MathieuColas 1998) a comme principe d'analyse de rapporter le fonctionnement des principales unités linguistiques à des phrases élémentaires. Dans ce modèle, il est postulé qu'une phrase élémentaire est constituée d'un prédicat et de ses éventuels arguments ${ }^{1}$ et que sa réalisation discursive résulte d'un double processus dit de linéarisation et d'actualisation (Gross et Vives 1986). Il s'ensuit que les unités linguistiques sont subdivisées principalement en trois catégories disjointes: celle des prédicats, celle des arguments élémentaires et celle des actualisateurs. Cette subdivision est fondée sur une hiérarchisation syntactico-sémantique: les prédicats prévalent sur les arguments et les actualisateurs sont subordonnés soit directement aux prédicats, soit aux relations entre ces derniers et leurs arguments respectifs.

La tête d'un groupe nominal étant le substantif en relation avec les autres constituants phrastiques majeurs, il est considéré que les autres éléments en constituent la détermination. Il en découle deux catégories majeures de déterminants: les prédéterminants et les modifieurs. Les premiers sont obligatoires et nécessairement en position frontale dans le groupe nominal, e.g. le dans le livre, les seconds sont forcément associés aux premiers ${ }^{2}$, e.g. beau dans le beau livre. L'inverse est inexact: les occurrences des premiers n'impliquent pas celles des seconds. Les modifieurs sont donc soit facultatifs soit obligatoires: on considère alors qu'il s'agit de modifieurs non liés dans le premier cas et de modifieurs liés dans le second cas $^{3}$.

Les prédéterminants sont clairement des actualisateurs étant donné qu’ils peuvent contribuer à la grammaticalisation de la phrase, i.e. à l'attestation discursive d'un prédicat éventuellement saturé par un ou plusieurs arguments (e.g. les dans Luc mange les bonbons). Si la détermination obligatoire participe de l'actualisation, elle peut néanmoins faire appel à des unités linguistiques qui ne sont pas nécessairement des actualisateurs: notamment les composantes adverbiale ou nominale de certains prédéterminants complexes (e.g. énormément de ou un tas de dans Luc mange (énormément de + un tas de) bonbons). Quant aux modifieurs facultatifs, ils ne sont pas directement assimilables à des actualisateurs car ils ne sont pas essentiels à la grammaticalisation de la phrase. De plus, en tant que constituants de déterminants complexes (cf. infra), ils sont de nature soit adjectivale (Luc mange les bonbons mentholés), soit nominale (Luc mange les bonbons de ce pâtissier), soit verbale (Luc mange les bonbons à sucer), i.e. des formes qui ressortissent principalement aux prédicats ou aux arguments, voire les deux quand les modifieurs sont de nature phrastique (Luc mange les bonbons qui ressemblent à des fraises).

Hors de toute considération pragmatique, il est généralement admis que le fonctionnement de la détermination implique la prise en compte des deux niveaux 
syntagmatiques suivants: le niveau interphrastique (J'ai rencontré Luc. Ce garçon est sympathique) et le niveau intraphrastique (J'ai rencontré un ami) ${ }^{4}$. Une autre distinction fondamentale caractérise la détermination: la détermination prédicative, d'une part, la détermination argumentale, d'autre part (Buvet, à paraître). Sa justification résulte de la hiérarchisation des unités linguistiques adoptée: les prédicats étant prépondérants par rapport aux arguments, leurs modes d'actualisation sont différents puisqu'ils ne se situent pas au même niveau structurel. Les déterminants obligatoires étant des actualisateurs des prédicats comme des arguments, ils se répartissent dans les deux catégories mentionnées. La première concerne les prédicats nominaux dans le cadre de constructions à support, la seconde porte sur les différents substantifs qui correspondent à des arguments. Indépendamment de la position hiérarchique des noms dans une phrase donnée, le fait qu'ils soient fondamentalement prédicatifs (e.g. bonté) ou bien élémentaires ${ }^{5}$ (e.g. pierre) est un autre facteur qui entre en ligne de compte dans la mesure où leur détermination peut s'interpréter différemment selon qu'ils appartiennent à l'une ou l'autre catégorie (Buvet, à paraître).

La détermination figée est une détermination complexe étant donné que le figement n'est concevable qu'au sein d'une séquence de mots (Gross 1996, Mejri 1997). Il s'agit cependant d'une condition nécessaire mais non suffisante pour faire état du caractère figé de la détermination. Avant de discuter des autres conditions, nous précisons la notion de détermination complexe.

\subsection{Typologie formelle de la détermination}

La figure ci-dessous correspond en grande partie à la classification de Gross (1996) des formes déterminatives:

FIGURE 1




La détermination simple concerne une partie des prédéterminants (e.g. trois dans Luc a acheté trois livres $)^{6}$. La détermination complexe se subdivise en deux catégories: la première concerne des associations de déterminants; la seconde se rapporte aux déterminants composés.

Pour ce qui est de la première catégorie, les séquences correspondent à la combinaison entre au moins deux prédéterminants ou bien un prédéterminant et un modifieur. L'association est soit libre, soit figée. Dans le premier cas de figure, la combinatoire est régie par les restrictions de sélection qui caractérisent les relations entre les déterminants associés dans un environnement phrastique donné prédéterminants varient en fonction de leurs valeurs respectives et selon un ordre préétabli dans Luc a lu (l'ensemble de + tous + E) $($ ces + les) (quelques + trois) livres. Dans le second cas de figure, le figement résulte du fonctionnement spécifique de l'association entre un prédéterminant et un modifieur donnés par rapport à un ou bien plusieurs substantifs, e.g. l'association entre l'article défini et le groupe prépositionnel $d u$ siècle relativement à affaire, contrat, coup... dans Luc a réalisé (l'/le) (affaire + contrat + coup $+\ldots) d u$ siècle, ou bien l'association de deux déterminants, e.g. l'association entre les prédéterminants un et ces par la préposition de dans Luc a une de ces chances; cf. infra.

Deux caractéristiques des séquences déterminatives de la seconde catégorie permettent de les distinguer clairement de celles de la première catégorie: (i) tous leurs constituants ne sont pas de nature déterminative; (ii) elles sont structurellement stables $^{8}$. La seconde caractéristique justifie l'appellation "détermination composée». On a affaire cependant à une autre condition nécessaire mais non suffisante du figement relatif à la détermination. En effet, l'attribut 'figé' est impropre pour une partie de la détermination composée car il s'agit de séquences déterminatives structurellement stables formées d'unités lexicales dont le sens n'est pas altéré par leur statut déterminatif': e.g. dans Luc a bu (beaucoup de + un litre de) vin, les déterminants complexes beaucoup de et un litre de ont toujours la même forme et les éléments beaucoup et litre conservent les valeurs qui leur sont propres en tant que, respectivement, adverbe et nom; cf. infra. Le critère sémantique apparaît donc primordial pour caractériser les déterminants composés figés, ces derniers ayant la particularité d'être structurellement stables mais sémantiquement atypiques. D’une façon générale, il appert que l'opacité sémantique est une propriété de première importance pour établir le caractère figé d'un déterminant. Ce point est traité plus en détail ci-dessous.

La Figure 1 ne permet pas de faire état de l'ensemble des situations qui se rapportent conjointement au figement et à la détermination. Il en existe trois sortes que nous présentons ci-dessous.

\section{La détermination du point de vue du figement}

La détermination et le figement sont impliqués simultanément dans des phénomènes linguistiques de trois façons différentes. La première concerne le statut des prédéterminants au sein des séquences figées, la deuxième a trait aux modifieurs et la dernière à la détermination complexe. 


\subsection{Les déterminants des séquences figées ${ }^{10}$}

Le premier type de situations n'est pas traité mais seulement évoqué ici car il se rapporte essentiellement à la question du figement et ne concerne qu'indirectement celle de la détermination étant donné, le plus souvent, que le principe de fonctionnement des déterminants n'est pas opératoire dans les séquences figées.

L'une des principales caractéristiques des expressions figées est d'être souvent sémantiquement non compositionnelles, i.e. elles ne s'interprètent pas directement en fonction des valeurs combinées de leurs constituants:

(1) Luc prend le bol

(2) Luc prend la tangente

Ces deux phrases, identiques sur le plan structurel, s'interprètent respectivement en fonction de quatre et deux constituants car la séquence du type V LE N est un idiotisme dans (2). Il s'ensuit que, contrairement à l'article défini de (1), le prédéterminant LE de (2) n'a pas un fonctionnement qui ressortit à l'anaphore ou à la deixis (Kleiber 1986) car il est dans la portée de figement de la locution verbale.

La question de la détermination n'est toutefois pas sans importance pour ce qui est des séquences figées. Tout d'abord, on observe qu'une variation déterminative donne parfois lieu à un idiotisme. Ainsi, le fait de remplacer le par un dans (1) conduit à deux interprétations de la phrase :

(1a) Luc prend un bol

La séquence prend un bol est soit libre, soit figée. Dans le premier cas de figure, le comportement de l'article indéfini est celui qui le caractérise dans ce type d'environnement phrastique ${ }^{11}$. Dans le second cas de figure, le fonctionnement de un est différent car ses propriétés sont annihilées, au moins partiellement, du fait que l'article est dans la portée de figement de la locution verbale. Par ailleurs, un grand nombre d'expressions figées présentent des variations locales, i.e. elles sont limitées à un seul de leurs constituants et n'ont aucun caractère régulier. Ces variations affectent, notamment, les déterminants (e.g. avoir (très + E) froid, faire (le + son) intéressant, mettre (certaines + des + les) formes).

L'interprétation du fonctionnement des prédéterminants dans les constructions libres n'a donc plus cours lorsque ces prédéterminants sont constitutifs d'expressions figées (e.g. lorsque la phrase Les carottes sont cuites est considérée au sens littéral, il est généralement admis que le déterminant défini contribue à l'identification du référent du nom carotte et cette interprétation de les est caduque dans la même phrase au sens figuré). La portée de cette observation n'est toutefois pas générale car elle est infirmée, notamment, par certaines phrases figées, e.g. le fonctionnement des différents déterminants n'est pas occulté par le figement dans La vérité sort de la bouche des enfants, car bien qu'il s'agisse d'une séquence figée, l'interprétation globale est compositionnelle ${ }^{12}$.

Les déterminants des expressions figées sont en rapport avec le figement d'une façon indirecte car s'ils se révèlent figés, c'est en tant qu'éléments de ces expressions. En général, les déterminants des expressions figées n'ont pas le fonctionnement qui les caractérise dans les séquences libres et il semble difficile de dégager des règles les concernant qui fassent un minimum d'abstraction de chaque situation. Il s'ensuit qu'il n'existe, selon nous, que deux catégories de déterminants figés: les séquences 
déterminatives qui ont le statut de modifieur et celles qui ressortissent à la détermination complexe. Nous les examinons successivement ci-dessous.

\subsection{Les modifieurs figés ${ }^{13}$}

Pour préciser le statut des modifieurs figés, deux aspects sont à prendre en compte: d'une part, il faut montrer qu'ils ne sont pas rattachés à un prédéterminant donné et, d'autre part, il convient de les distinguer des locutions adjectivales. Le premier point a pour conséquence que les modifieurs figés ne donnent pas lieu à une détermination figée et le second qu'ils s'apparentent à des compléments du nom. Dans un premier temps, nous indiquons comment sont configurées les séquences déterminatives étudiées puis nous constatons qu'il s'agit de modifieurs non liés. Dans un deuxième temps, nous discutons des deux catégories de modifieurs suivantes: les adjectifs épithètes et les compléments du nom afin d'établir que les modifieurs figés se rapportent à la seconde catégorie puis nous discutons de leur caractère figé.

Les séquences déterminatives figées recensées comportent, entre autres, des groupes prépositionnels: le tableau ci-dessous répertorie les prépositions les plus fréquentes en position initiale.

TABLEAU 2

\begin{tabular}{|l|l|}
\hline PREP & Exemple \\
\hline$\grave{a}$ & (une conversion) à bâtons rompus \\
\hline$d e$ & (des secours) de première urgence \\
\hline en & (une réception) en grand tralala \\
\hline jusque & (des amis) jusqu'à la bourse \\
\hline sous & (un examen) sous toutes les coutures \\
\hline sur & (des confidences) sur l'oreiller \\
\hline
\end{tabular}

Les prépositions sont toujours suivies d'un nom ou d'un groupe nominal, à l'exception de $a$ et $d e$ qui autorisent aussi respectivement un verbe à l'infinitif (un homme à abattre) et un adverbe (un homme de peu). Les groupes prépositionnels ainsi formés correspondent à des modifieurs qui se combinent avec divers prédéterminants pour constituer la détermination d'un substantif donné:

$$
\begin{aligned}
& \text { (une }+ \text { plusieurs }+ \text { la }+\ldots) \text { conversion }(s) \text { à bâtons rompus } \\
& (\text { des }+ \text { les }) \text { secours de première urgence } \\
& (\text { une }+ \text { plusieurs }+ \text { la }) \text { réception }(s) \text { en grand tralala } \\
& (\text { des }+ \text { les }) \text { amis jusqu'à la bourse } \\
& (\text { un }+ \text { plusieurs }+ \text { l' }+\ldots) \text { examen }(s) \text { sous toutes les coutures } \\
& (\text { des }+ \text { plusieurs }+ \text { les }) \text { confidences sur l'oreiller }{ }^{14}
\end{aligned}
$$

Rappelons que les modifieurs liés ont la particularité d'être obligatoires et ils peuvent donner lieu à une détermination figée lorsqu'ils dépendent d'un seul déterminant ou type de déterminant (cf. infra). Les groupes prépositionnels pris en compte dans cette section pouvant se rattacher à des prédéterminants variés, il s'agit donc de modifieurs non liés. Ce point étant précisé, il convient à présent de préciser leur nature.

Les modifieurs figés ne correspondent pas à des adjectifs composés épithètes (un homme d'un âge canonique) mais à des compléments du nom (un homme d'un village 
voisin $)^{15}$. Rappelons que les seconds ont en commun avec une partie des premiers d'être des groupes prépositionnels mais qu'ils sont incompatibles avec la position attribut et la pronominalisation en le qui caractérisent les seuls adjectifs prédicatifs (cf. Gross 1996) :

(3) Cet homme d'un âge canonique (adjectif composé)

(3a) Cet homme est d'un âge canonique

(3b) D'un âge canonique, cet autre homme l'est aussi

(4) Cet homme d'un village voisin (complément du nom)

(4a) ${ }^{*}$ Cet homme est d'un village voisin

(4b) *D'un village voisin, cet autre homme l'est aussi

Les modifieurs figés sont également incompatibles avec ces deux propriétés:

(5) Ces chaussettes en accordéon (modifieur figé)

(5a) ${ }^{*}$ Ces chaussettes sont en accordéon

(5b) ${ }^{\star}$ En accordéon, ces chaussettes le sont aussi (modifieur figé)

Ne fonctionnant pas également comme des adjectifs épithètes et étant du même type configurationnel que les compléments du nom, les modifieurs figés sont assimilés à ces derniers. Les agrammaticalités des types (4a), (4b) et (5a), (5b) justifient ce rapprochement. Pour autant, les modifieurs figés sont atypiques en tant que compléments du nom, car contrairement aux modifieurs libres de la même catégorie (cf. Buvet 2002a), les relations entre leurs principaux constituants et la tête nominale qu'ils déterminent sont difficilement analysables. D’une façon générale, le caractère figé des modifieurs non liés est imputable aux faits suivants qui sont autant de critères de figement (la séquence à cent balles est prise comme exemple de modifieur figé):

(i) ce sont des séquences structurellement stables et globalement invariantes:

une question $\left(\grave{a}+{ }^{*} d e+{ }^{*} e n+{ }^{*}\right.$ sous $+{ }^{*}$ sur $)$ cent balles

une question à (cent $+{ }^{*}$ dix $+{ }^{*}$ mille) balles

une question à cent (balles $+{ }^{*}$ euros $+{ }^{*}$ francs)

(ii) les groupes prépositionnels sont le plus souvent sémantiquement opaques, i.e. leur relation avec le nom déterminé est inanalysable:

${ }^{*}$ cette question coûte cent balles

${ }^{*}$ cette question vaut cent balles

*le prix de cette question est de cent balles

(iii) leur distribution gauche est généralement restreinte à un seul substantif:

une $\left({ }^{*}\right.$ demande $+{ }^{*}$ devinette + question $+{ }^{*}$ réponse $)$ à cent balles

Pour ce qui est des critères (ii) et (iii), il y a des exceptions:

- la relation entre le modifieur et la tête nominale est parfois analysable

un nez en trompette

ce nez est en forme de trompette

ce nez a l'air d'une trompette

- plus d'un substantif peut avoir comme expansion le même modifieur figé:

un patron de choc

un tandem de choc 
Il est question à présent de modifieurs liés. Bien que leurs caractéristiques internes soient parfois proches des modifieurs non liés figés, c'est secondaire du point de vue du figement car les modifieurs liés ne sont considérés comme figés qu'en raison de l'une de leurs caractéristiques externes, ils se rattachent à un seul prédéterminant ou type de prédéterminant.

\subsection{La détermination figée}

Les déterminants à caractère figé examinés précédemment nécessitent d'être associés à d'autres déterminants pour constituer une détermination, tandis qu'à présent il est question de détermination, i.e. de séquences déterminatives dont la combinatoire avec des noms donnent lieu directement à la formation de groupes nominaux. Nous présentons dans un premier temps les associations figées de déterminants puis dans un deuxième temps les prédéterminants composés figés.

\subsubsection{Associations figées de déterminants}

Il s'agit essentiellement de séquences constituées, d'une part, toujours du même prédéterminant ou bien du même type de prédéterminant et, d'autre part, d'un modifieur lié, les séquences étant disjointes sauf quand les modifieurs correspondent à des adjectifs épithètes antéposés. Il y a également quelques suites formées d'une séquence déterminative antéposée et d'un prédéterminant ainsi qu'une combinatoire de prédéterminants dont la valeur est atypique.

\subsubsection{Prédéterminant associé à un modifieur}

Après avoir présenté les principales configurations de ce type d'association figée de déterminants, nous établissons en quoi le figement affecte ces séquences déterminatives puis nous les étudions du point de vue de l'opposition entre la détermination prédicative et la détermination argumentale.

Les modifieurs liés sont de toutes sortes: il s'agit notamment de groupes prépositionnels et d'adjectifs. Les associations recensées ont un caractère figé parce que l'on a affaire à des modifieurs qui constituent avec le prédéterminant une configuration unique dont la valeur globale correspond à une signification grammaticale (cf. infra). Dans de telles configurations, la position prédéterminant est occupée par un seul déterminant ou type de déterminant qui varie d'une configuration à l'autre.

Précisons que tous les modifieurs liés ne donnent pas lieu à une détermination figée, par exemple certains sont des modifieurs obligatoires ayant trait à la prédication seconde, i.e. sans rapport avec la question du figement (Buvet, à paraître; Buvet 2002b; Riegel 1988):

(6) Luc a une allure $\left({ }^{\star} E+\right.$ décontractée $)$

(7) Luc a un caractère $\left({ }^{\star} E+\right.$ facile $)$

Faute de place, les différentes configurations répertoriées sont seulement évoquées. Nous indiquons dans le tableau suivant différents types d'association figée en fonction des principaux prédéterminants, puis nous présentons les principales particularités des modifieurs. Nous établissons ensuite en quoi ces différentes sortes de déterminations sont figées. 
TABLEAU 3

\begin{tabular}{|l|l|l|}
\hline type d'association figée & occurrence & Exemple \\
\hline DNUM_MODIF & DNUM_et des poussières & Il y a vingt ans et des poussières \\
\hline DU_MODIF & DU_àvolonté & Luc propose du pain à volonté \\
\hline LE_MODIF & LE_au beau fixe & Luc a le moral au beau fixe \\
\hline UN_MODIF & UN_de cabri & Luc fait des bonds de cabri \\
\hline ZERO MODIF & ZERO mauvaise & Luc a mauvaise mine \\
\hline ZERO_MODIF & $\begin{array}{l}\text { ZERO_jusqu'au bout des } \\
\text { ongles }\end{array}$ & $\begin{array}{l}\text { Luc est peintre jusqu'au bout des } \\
\text { ongles }\end{array}$ \\
\hline
\end{tabular}

Le Tableau 3 ci-dessus ne rend compte que partiellement des phénomènes étudiés puisqu'il occulte plusieurs sous-types d'associations figées, e.g. les configurations telles que le prédéterminant est obligatoirement:

- l’article défini singulier:

(7) Luc a $\left(\right.$ la $+{ }^{*}$ les $)$ conscience large

- l'article indéfini pluriel:

(8) Luc a (des $+{ }^{*}$ un) ennuis à tire-larigot

Par ailleurs, il existe des situations intermédiaires entre la détermination figée et la détermination libre issue de la combinatoire entre des modifieurs figés et des prédéterminants variés: différents modifieurs peuvent se rattacher à plus d'un type de prédéterminant mais ne sont pas compatibles avec tous. Les fortes restrictions sur les prédéterminants impliquent que l'on a affaire à des modifieurs liés. Les séquences déterminatives qui en résultent ont donc les mêmes caractéristiques que les autres associations figées de déterminants, nonobstant la variété limitée des prédéterminants, ce sont des configurations où les prédéterminants sont (i) soit divers quantifieurs, soit des articles indéfinis, (ii) soit le partitif, soit l'article indéfini pluriel. On peut donc compléter le Tableau 3 comme suit:

TABLEAU 3A

\begin{tabular}{|l|l|l|}
\hline type d'association figée & occurrence & exemple \\
\hline (DQUA+UN)_MODIF & (DQUA+UN) de cet acabit & $\begin{array}{l}\text { Luc a rencontré (plusieurs } \\
\text { joueurs }+ \text { un joueur) de cet } \\
\text { acabit }\end{array}$ \\
\hline (DU+des)_MODIF & $\begin{array}{l}\text { Luc a (du travail +des soucis) } a ̀ ~ \\
\text { la pelle }\end{array}$ \\
\hline
\end{tabular}

Bien que les déterminations du Tableau 3a ne soient pas des configurations spécifiques, la position frontale étant occupée par plus d'un type de prédéterminant, elles sont cependant indéniablement figées dans la mesure où la variété se limite à deux types. De plus, dans le cas de l'association du type (DU+des)_MODIF, les corrélations morphologiques entre des et $d u$, l'un et l'autre prédéterminants sont formés de la préposition de et de l'article défini, font que cette dualité est atténuée et, par conséquent, le caractère figé de la séquence est renforcé. 
Les modifieurs liés impliqués dans une détermination figée sont de quatre sortes:

a) des adjectifs simples, e.g. carabiné:

(9) Luc a une fièvre carabinée des groupes prépositionnels, e.g. de singe

(10) Luc a bu un vin de singe des relatives, e.g. qui tue:

(11) Léa a un regard qui tue

b) des séquences introduites par une conjonction, e.g. comme s'il en pleuvait:

(12) Luc recevait des coups comme s'il en pleuvait

c) des infinitives:

(13) Luc nous raconte des histoires à faire peur

Les modifieurs du type (a) prouvent que le figement porte ici sur la relation entre le prédéterminant et son expansion car, l'un et l'autre étant des formes simples, la condition de polylexicalité afférente au figement (Gross 1996) ne peut être satisfaite que dans le cadre de cette relation. Le rattachement du modifieur à un prédéterminant unique constitue donc une détermination dont le caractère figé résulte, entre autres, du fait que la séquence déterminative disjointe est une configuration stable. Il s'ensuit, quels que soient les modifieurs liés, que le figement affecte la relation avec le prédéterminant et son expansion, indépendamment de la forme que recouvre cette dernière.

Les modifieurs liés du type (b) ont des configurations beaucoup plus diversifiées que celles des modifieurs non liés figés. Ils ont néanmoins les mêmes caractéristiques: ce sont des séquences structurellement stables qui admettent éventuellement des variations limitées à un seul de leurs constituants (e.g. Luc a un menton à (double + triple) étage) et, d'une façon générale, sont sémantiquement opaques, d'une part, distributionnellement restreintes, d'autre part ${ }^{16}$. Toutefois, ces diverses particularités n'expliquent qu'en partie que l'on ait affaire à une détermination figée, un autre élément d'explication est le caractère unique de la relation entre un prédéterminant et un modifieur lié.

Les modifieurs liés des types (c) et (d) sont moins fréquents que les deux premiers. Bien qu'il ne s'agisse pas de groupes prépositionnels, ce sont des séquences qui ont des caractéristiques comparables et donnent également lieu à une détermination figée.

Le dysfonctionnement sémantique qui caractérise les modifieurs liés complexes, i.e. ils n'ont pas un sens compositionnel, concerne également les modifieurs liés simples, du type adjectival, dans la mesure où ces derniers sont sémantiquement atypiques. Ainsi, il est clair que l'interprétation de l'adjectif n'est pas comparable dans les énoncés suivants :

(14) Luc a une démarche souple

(15) Luc a une peur bleue

Il est prédicatif uniquement dans le premier cas:

(14a) La démarche de Luc est souple

(15a) ${ }^{\star}$ La peur de Luc est bleue 
L'interprétation de souple dans (14) relève du sens lexical alors que celle de bleue dans (15) procède du sens grammatical (cf. infra). Dans les deux cas, l'adjectif fonctionne comme un modifieur lié puisqu'il est obligatoire:

(14b) Luc a une démarche $\left({ }^{\star} E+\right.$ souple)

(15b) Luc a une peur $\left({ }^{*} E+\right.$ bleue $)$

Cependant, le caractère obligatoire de souple relève de la prédication seconde dans (14) tandis que bleue, lorsqu'il se combine avec une, relativement à peur, correspond à une détermination figée dans (15):

(14c) Luc a (la +ma+une) démarche souple

(15c) Luc a $\left({ }^{*} l a+{ }^{*} m a+\right.$ une) peur bleue

Le caractère sémantiquement opaque de l'adjectif corrobore cette interprétation de la séquence déterminative une_bleue.

L'association figée entre un prédéterminant et un modifieur correspond aussi bien à une détermination prédicative qu'à une détermination argumentale. La première catégorie de détermination implique que l'on ait affaire à des constructions à support:

- construction en avoir

(16) Luc avait une faim de loup

- construction en avoir lieu

(17) Une bataille rangée a eu lieu

- construction en donner

(18) Léa a donné à Luc un baiser de nourrice

- construction en éprouver

(19) Luc éprouve un amour fou pour Léa

- construction en mener

(20) Luc mène grande vie ${ }^{17}$

Toutes les constructions en avoir qui sont le cadre d'une détermination figée ne sont pas nécessairement à verbe support dans la mesure où avoir peut-être un relateur:

(21) Luc a des yeux en vrille

Il s'agit de situations proches de celles où le modifieur obligatoire est un adjectif épithète (Buvet, à paraître) :

(22) Luc a des yeux bleus

Néanmoins, (21) diffère de (22) du fait que:

(i) l'expansion n'est pas possible en position prédicative:

(21a) $?^{\star}$ Les yeux de Luc sont en vrille

(22a) Les yeux de Luc sont bleus

(ii) toute variation du prédéterminant de la séquence déterminative est interdite:

(21b) Luc a (des + ?*$^{*}$ mes + ?*les) yeux en vrille

(22b) ? Luc a (des + mes + les) yeux bleus

Il s'ensuit le caractère figé de la suite disjointe une_en vrille dans (21). 
Le fait que le substantif yeux est un nom élémentaire du type méronymique justifie que l'on interprète avoir comme un relateur (cf. ibidem). La plupart des noms élémentaires qui ont une détermination figée correspondant à l'association d'un prédéterminant et d'un modifieur sont des noms de < partie du corps $>$ au sein de constructions en avoir. Les quelques autres noms élémentaires ont une détermination figée du type argumental:

(23) Luc possède un chien à sa mémère ${ }^{18}$

De l'étude du corpus constitué, il ressort que la détermination argumentale concerne surtout des noms prédicatifs:

(24) La police a attrapé un escroc de haut vol

L'une ou l'autre détermination, lorsqu'elle est figée et constituée d'un prédéterminant ou d'un type de prédéterminant et d'un modifieur, est telle que l'expansion est souvent sémantiquement opaque comme l'illustrent les séquences à sa mémère et de haut vol des exemples (23) et (24). Par contre, leur combinatoire avec les noms fait apparaître une différence entre les deux catégories de détermination car, le plus souvent, la détermination prédicative concerne un seul substantif, voire quelques uns:

(25) Luc a poussé des (cris $+{ }^{*}$ hurlements) d'orfraie

(26) Luc a ressenti un (e) ( ${ }^{*}$ allégresse + bonheur + joie) sans mélange

alors que la détermination argumentale est plus hétérogène puisqu’elle peut avoir trait aussi bien à :

- un seul substantif

(27) Luc m'a dit un secret de Polichinelle

- plusieurs substantifs

(28) Le restaurant sert ((des (apéritifs + des boissons alcoolisés $+\ldots)+d u$

$($ champagne + vin rouge...+$))$ à discrétion

- toutes sortes de substantifs:

(29) Luc a imaginé des (programmes + projets + solutions + théories $+\ldots$ ) à foison

La divergence entre la détermination prédicative et la détermination argumentale proviendrait des particularités mêmes de la première. Une construction à support est telle, d'une part, qu'elle permet de faire état de la saturation d'un prédicat nominal par en général au moins un argument dans un cadre phrastique et, d'autre part, qu'une partie de la nature des autres constituants de la phrase, les actualisateurs, est subordonnée à celle du nom prédicatif. Il en est ainsi des verbes supports (e.g. Luc (donne $+{ }^{*}$ fait) une claque à Max; Luc ( ${ }^{*}$ donne + fait) un hold-up) et de la détermination prédicative qui peut être extrêmement contrainte dans le cadre d'une même classe sémantique de substantifs (cf. Buvet 1998). La dépendance des actualisateurs au prédicat nominal est encore plus marquée lorsqu'ils sont porteurs d'une signification grammaticale (cf. infra) comme l'ont montré de nombreuses observations sur les verbes supports (Luc donne des conseils à Max, Luc multiplie les conseils à Max, Luc abreuve Max de conseils, ${ }^{\star}$ Luc flanque des conseils à Max; Luc donne des gifles à Max, ${ }^{\star}$ Luc multiplie les gifles à Max, ${ }^{*}$ Luc abreuve de gifles Max, Luc flanque des gifles à Max) et il en est de même pour la prédication déterminative qu'elle soit libre ou figée (Luc éprouve de l'amitié pour Max, Luc éprouve une amitié sincère pour Max, Luc 
éprouve une amitié particulière pour Max, ${ }^{\star}$ Luc éprouve une amitié féroce pour Max, ${ }^{\star}$ Luc éprouve une amitié terrible pour Max, Luc éprouve de la haine pour Max, ${ }^{\star}$ Luc éprouve une haine sincère pour Max, ${ }^{\star}$ Luc éprouve une haine particulière pour Max, Luc éprouve une haine féroce pour Max, Luc éprouve une haine terrible pour Max).

La détermination argumentale se distingue de la détermination prédicative du fait que sa nature dépend, à la fois de celle du nom en position d'argument et de la relation de ce dernier avec le prédicat auquel il est rattaché. Lorsqu'elle est principalement imputable aux particularités du substantif, la détermination figée du type argumental est très souvent distributionnellement restreinte:

(30) Luc navigue sur ( ${ }^{*}$ un lac + une mer) d'huile

Quand elle ressortit essentiellement au prédicat, cette détermination peut concerner une plus grande variété de substantifs (cf. Buvet, à paraître):

(31) Luc a vendu des (chapeaux + projets + tests $+\ldots)$ à la folie

La séquence déterminative des_à la folie a 'une grande quantité' comme signification grammaticale: elle caractérise la relation entre le prédicat et son deuxième argument indépendamment de la nature de celui-ci. Il s'ensuit que cette détermination est admise par tous les substantifs du domaine d'arguments relatif à N1 du prédicat vendre.

Nous examinons à présent des associations figées de déterminants dont les caractéristiques sont différentes de celles que nous venons d'étudier.

\section{Autres associations figées de déterminants}

Trois sortes d'associations figées de déterminants sont relativement atypiques. Si toutes les trois concernent des séquences déterminatives constituées d'une façon similaire, les deux premières se différencient de la dernière par les propriétés du constituant antéposé et, par ailleurs, se distinguent entre elles par la signification grammaticale de la détermination: la première implique une valeur quantitative, la seconde une valeur intensive. Nous étudions successivement chaque sorte d'association.

Précisons tout d'abord que les séquences déterminatives prises en compte ici sont en nombre limité, qu'elles ont la particularité d'être globalement des prédéterminants et, à une exception près, de correspondre à des constructions formées de deux constituants déterminatifs reliés par la préposition $d e$ : le premier est toujours en position frontale dans un groupe nominal, le deuxième est nécessairement et exclusivement soit du type DDEF (i.e. un prédéterminant défini) soit un générique du type LE:

(32) Luc a lu la plupart des livres

(33) Luc passe le plus clair de son temps à dormir

(34) Agir ainsi est le comble de la bêtise

(35) Se comporter de la sorte est le summum de la goujaterie

(36) Luc a attrapé une de ces migraine.

Les premiers constituants des séquences déterminatives figées de (32) à (35) ont en commun, d'une part, leur configuration, ils sont formés de le suivi d'un élément ou d'une suite d'éléments ayant plus ou moins trait à la quasi-totalité ou au haut degré et, d'autre part, de ne pas pouvoir se combiner directement avec un substantif pour former un groupe nominal: 
(32a) * Luc a lu la plupart de livres

(33a) ${ }^{*}$ Luc passe le plus clair de temps à dormir

(34a) * Agir ainsi est le comble de bêtise

(35a) * Se comporter de la sorte est le summum de goujaterie

Il existe néanmoins des différences significatives entre les séquences déterminatives de (32) et (33) et celles de (34) et (35). Nous ne tenons compte pour l'instant que des associations dont font état les deux premiers exemples.

Les associations figées la plupart de DDEF et le plus clair de POSS

Si l'absence des seconds constituants donne lieu aux phrases inacceptables (31a) et (32a), celle des premiers constituants de (30) et (31) est concevable nonobstant la perte de sens qui résulte de leur effacement:

(32b) Luc a lu les livres

(33b) Luc passe son temps à dormir

Les constituants la plupart et le plus clair ont des propriétés syntaxiques très différentes:

- le premier s'associe, par le biais de la préposition de, avec les différents déterminants définis, le second uniquement avec le déterminant possessif ${ }^{19}$ :

(32c) Luc a lu la plupart (des + de ces + de mes) livres

(33c) Luc passe le plus clair $\left({ }^{*} d u+{ }^{*} d e c e+\right.$ de son) temps à dormir

- contrairement à le plus clair, la plupart peut également fonctionner comme un pronom aussi bien en position sujet qu'en position objet:

(32d) Les livres, la plupart ont été lus par Luc

(32e) Les livres, Luc en a lu la plupart

(33d) * Son temps, Luc en passe le plus clair à dormir ${ }^{20}$

- la séquence déterminative comprenant la plupart se rapporte à toutes sortes de substantifs, celle qui comporte le plus clair se combine quasi exclusivement avec le nom temps $s^{21}$; de plus, dans le premier cas, les GN s'observent dans les diverses positions syntaxiques et fonctionnent aussi bien en position argumentale qu'en position prédicative (La plupart des voitures roulent au ralenti, La police a contrôlé la plupart des voitures, Luc a arraché des pages de la plupart de mes livres, Luc a fait la plupart des betises, etc.) tandis que, dans le second cas, le GN est obligatoirement en position prédicative au sein de la construction à support passer ou l'une des variantes de ce verbe

(33e) Luc (consacre + occupe + passe + prend) le plus clair $\left({ }^{*} d u+\right.$ de ce + de son) temps à dormir ${ }^{22}$

D’autres formes déterminatives ont un fonctionnement similaire à la plupart: (i) elles ne peuvent pas se combiner directement avec un substantif; (ii) elles précèdent obligatoirement des définis par l'intermédiaire de la préposition de; (iii) elles sont acceptées par toutes sortes de substantifs; (iv) les GN où elles figurent occupent des positions syntaxiques variées; (v) sémantiquement, elles correspondent à des marqueurs de quantité. Les particularités (i) et (ii) justifient qu'on les considère en tant que premier constituant d'associations figées de déterminants: 
(36) Luc a lu la totalité $\left({ }^{*} E+(\right.$ des + de ces + de mes $\left.)\right)$ livres

(37) Luc a lu une $(E+$ bonne + petite + grande $)$ partie $\left({ }^{*} E+(\right.$ des + de ces + de mes $\left.)\right)$ livres

En tant que déterminant, la forme tout a deux fonctionnements différents (cf. Buvet, à paraître; Kleiber et Martin 1977) : celui de prédéterminant (Tout homme est mortel) et celui d'élément déterminatif en position frontale dans un GN nécessairement antéposé à un prédéterminant (J'ai mangé tout $\left({ }^{\star} E+l e\right)$ gâteau). Il en résulte au moins deux emplois de tout, le deuxième ayant des formes relatives aux catégories du genre et du nombre, contrairement au premier qui a trait uniquement à la catégorie du genre. Le deuxième emploi, par son sens et les particularités de sa combinatoire, s'apparente aux séquences du type la plupart malgré les différences suivantes: (i) il a une forme simple sujette à variation; (ii) il ne nécessite pas la préposition de pour être associé à un prédéterminant; (iii) il ne se combine pas exclusivement avec des définis:

(38) Luc a lu tout $(c e+l e+$ mon + un) livre

Le point (iii) interdirait d'interpréter tout comme premier constituant d'une association figée; néanmoins, étant donné que les prédéterminants qui se combinent avec tout sont restreints à un et les différents types de définis, on peut considérer les séquences déterminatives du type TOUT (DDEF + un $(\boldsymbol{e}))$ comme ressortissant au figement, à l'instar des séquences du type (DQUA+UN)_MODIF ou (DU+des)_MODIF qui se caractérisent par la variété limitée de l'un de leurs constituants (cf. supra).

Si le plus clair n'est pas commutable avec les constituants du type la plupart ainsi que tout dans toutes sortes d'environnements, ces autres constituants peuvent commuter avec cette séquence dans (33):

(33f) Luc passe (la plupart de + la totalité de + une $(E+$ bonne + petite + grande) partie de + tout le plus clair de son) temps à dormir

Les associations de déterminants dont les premiers constituants sont le comble ou le summum fonctionnent différemment de celles dont les premiers constituants sont la plupart ou le plus clair tant sur le plan syntaxique que du point de vue sémantique.

- Les associations figées du type le (comble + summum) de le:

Aussi bien pour (34) que pour (35), on constate que l'effacement du premier constituant n'entraîne pas celui de la préposition $d e$ :

(34b) Agir ainsi est $\left(d e+{ }^{*} E\right)$ la bêtise

(35b) Se comporter de la sorte est $\left(\right.$ de $+{ }^{*} E$ ) la goujaterie

On ne peut pas pour autant en déduire que de la dans (34) et (35) est un article partitif auquel serait respectivement accolé, en antéposition, les constituants le comble et le summum. Plusieurs propriétés contreviennent à cette déduction car elles font état de différences entre l'article partitif et les séquences le comble de la et le summum de la:

1) permutation des séquences verbale et nominale placées de part et d'autre de la copule:

(34c) $\left({ }^{*}\right.$ De la + Le comble de la) bêtise est d'agir ainsi

(35c) $\left({ }^{*}\right.$ De la + Le summum de la) goujaterie est de se comporter de la sorte 
Selon Riegel (1985), les conditions de la permutation dans une construction en être exigent généralement que les GN déplacés en tête de phrase correspondent à des expressions définies; par conséquent, dans (34c) et (35c), le caractère non défini du prédéterminant de la expliquerait les inacceptabilités qui l'affectent et, inversement, les acceptabilités relatives aux séquences déterminatives le comble de la et le summum de la justifieraient leur caractère défini.

- Dislocation de la suite nominale ou verbale avec reprise par ce:

(34d) Agir ainsi, c'est ( ${ }^{*}$ de la + le comble de la) bêtise

(35d) Se comporter de la sorte, c'est $\left(^{*}\right.$ de la + le summum de la) goujaterie

(34e) $\left({ }^{\star}\right.$ De la + Le comble de la) bêtise, c'est d'agir ainsi

(35e) $\left({ }^{\star}\right.$ De la + Le summum de la) goujaterie, c'est de se comporter de la sorte

2) effacement de la tête nominale:

(34f) [en matière de bêtise] Agir ainsi est le comble

(35f) [en matière de goujaterie] Se comporter de la sorte est le summum

Cette dernière propriété ne concerne en aucun cas le partitif de la puisque son statut d'article procède de sa combinatoire obligatoire avec un substantif et, par ailleurs, la propriété 3) peut se combiner avec la propriété 2); il s'ensuit:

3) effacement de la tête nominale et dislocation de la suite verbale avec reprise parce:

(34g) [en matière de bêtise] Le comble, c'est d'agir ainsi

(35g) [en matière de goujaterie] Le summum, c'est de se comporter de la sorte ${ }^{23}$

Le fonctionnement différent des GN uniquement à article partitif et des GN comportant en position frontale le comble ou le summum explique leurs comportements divergents en regard de ces quatre propriétés. Dans le premier cas, les GN de (34b) et (35b) stipulent que l'on a affaire à une occurrence non spécifiée de l'état dénoté respectivement par les noms abstraits bêtise ou goujaterie ${ }^{24}$. Les propriétés 1) et 2) prouvent l'asymétrie de la relation entre la suite verbale et la suite nominale, au sein de la construction en être, la seconde contribuant à caractériser la première $e^{25}$. Dans le second cas, au contraire, les GN de (34) et (35) sont relatifs à une occurrence spécifique de l'état mentionné. Si chaque suite nominale permet de caractériser chaque suite verbale, en établissant l'équivalence pour chaque paire de suites, les propriétés 1) et 2) montrent que cette caractérisation n'implique pas une relation d'élément à ensemble ${ }^{26}$ entre leurs dénotations respectives mais plutôt une relation de similitude et, par conséquent, que l'occurrence spécifique à laquelle se rapporte le GN correspond à la dénotation de l'infinitive; les formulations suivantes le montrent également:

(34h) Qu'est-ce que c'est le comble de la bêtise? Agir ainsi

(35h) Qu'est-ce que c'est qu'agir ainsi?

Le comble de la bêtise

Parmi les facteurs qui expliquent la correspondance de cette équivalence, il y a le caractère défini du GN et la condition d'unicité qui lui est rattachée (cf. Riegel et al. 1994), ces deux points étant mis en évidence par l'absence de commutation entre le et $u n$ en position frontale dans le GN: 
(34i) Agir ainsi est (le $\left.+{ }^{*} u n\right)$ comble de la bêtise

(35i) Se comporter de la sorte est (le $+{ }^{*}$ un) summum de la goujaterie

Les phénomènes déterminatifs, dont font état (34) et (35), et la relative autonomie des constituants le comble et le summum, qu'attestent les propriétés 3) et 4), s'observent uniquement au sein d'une construction en être, mais la construction n'a pas un caractère figé pour autant, le seul GN pouvant faire l'objet d'une reprise:

(34j) Agir ainsi est le comble de la bêtise. Cette bêtise lui a d'ailleurs valu les pires ennuis

(35j) Se comporter de la sorte est le summum de la goujaterie. Cette goujaterie est impardonnable

Les seules occurrences de le comble et le summum, au sein d'une construction en être, s'observent dans une séquence déterminative, il s'agit donc clairement de déterminants. La possibilité de les détacher de l'ensemble de la séquence prouve que la forme de n'est pas un élément de l'article partitif, en tant que prédéterminant composé, mais bien un relateur comme dans les séquences déterminatives de (32) et (33). Par conséquent, le comble ou le summum et la forme la constituent, par le biais de la préposition $d e$, une association de déterminants telle que le constituant postposé est nécessairement du type générique. Pour établir le dernier point, il convient de prendre en compte la nature des constituants antéposés.

Les particularités sémantiques de comble et summum dans les phrases en être justifient la seule présence de le à leur gauche ${ }^{27}$ : ces formes indiquant le plus haut point relativement à un état dénoté par un nom abstrait du type bêtise ou goujaterie, une seule occurrence de l'état stipulé est concevable. Il en résulte, d'une part, que l'interprétation du déterminant interne le (i.e. il relève du niveau infra-phrastique [cf. Buvet 2003b]) s'apparente à celle d'un défini autonome, un prédéterminant dont la combinatoire avec un substantif donne lieu à un GN uni-référentiel ${ }^{28}$ (Corblin 1987), d'autre part, que l'interprétation partitive des GN avec la plupart et le plus clair en position frontale n'est pas effective pour les GN avec le comble et le summum en position frontale dans (34) et (35). Si l'association de la plupart avec les dans (32) par le biais de la préposition de et celle de le plus clair avec son par le même biais dans (33) donnent lieu à des GN correspondant, sur le plan quantitatif, à des sous-ensembles respectifs aux ensembles relatifs aux GN définis les livres et son temps, en revanche, une telle interprétation est impossible dans (34) et (35): il est exclu de rapporter les relations entre le comble de la bêtise et la bêtise, d'une part, entre le summum de la goujaterie et la goujaterie, d'autre part, à des relations de sous-ensembles à ensembles car les GN du type LE $\mathbf{N}$ ne se prêtent pas à une caractérisation quantitative étant donné les traits abstrait et massif des noms bêtise et goujaterie au sein de la construction en être. Par contre, ils autorisent une lecture générique telle qu'il est question de l'ensemble des occurrences relatives aux états stipulés respectivement par les noms bêtise et goujaterie (Kleiber 1990). Par conséquent, l'association des constituants le comble et le summum avec le générique la par le biais de la préposition de permet de stipuler qu'il s'agit d'une occurrence spécifique, celle qui a trait à l'état porté au plus haut point. Un tel état étant d'une façon générale difficilement discernable, l'association de déterminants est relativement opaque sur le plan sémantique; c'est en fait une intensité forte ${ }^{29}$ qui est signifiée avec la détermination complexe. L’opacité sémantique de ce type d'association de déterminants est révélatrice de son caractère figé. 
Des analyses ci-dessus, il ressort que les séquences déterminatives le comble de LE et le summum de LE ne peuvent s'observer qu'au sein d'une construction en être dans la mesure où en contribuant à la caractérisation d'états du type bêtise ou goujaterie elles forment avec les substantifs qui dénomment les états des GN qui qualifient, par le biais de la copule être, une activité, un événement ou une situation spécifiés par des suites verbales ou nominales ${ }^{30}$. Les suites reliées par être sont alors généralement sémantiquement contraintes: la nature du prédicat de la suite verbale ou nominale qualifiée par le GN en le comble ou le summum et celle de la tête nominale de ce dernier sont interdépendantes:

(39) Le comble de la sottise est de tout (ignorer + ? comprendre)

(40) Le summum de l'intelligence est de tout (?ignorer + comprendre)

Signalons enfin que d'autres formes que comble et summum ont un fonctionnement assez similaire en tant qu'élément déterminatif:

(41) Tuer son frère est le paroxysme de la violence

Par rapport aux séquences déterminatives examinées jusqu’à présent, l'association figée de déterminants un de ces s'avère particulière à plus d'un titre.

\section{L'association figée un de ces}

Si la configuration de la séquence un de ces présente des similitudes avec celles des autres associations figées de déterminants, on observe néanmoins des différences majeures entre cette association et les précédentes.

En premier lieu, les deux constituants déterminatifs reliés par la préposition de ont la particularité d'être l'un et l'autre des prédéterminants. Il s'ensuit, en ce qui concerne (36), qu'aucun constituant ne donne lieu à une phrase inacceptable par son absence :

(36a) Luc a attrapé une migraine

(36b) Luc a attrapé ces migraines

Pour décrire l'association de déterminants, contrairement à ce qui a été mentionné à propos des précédentes, l'effacement du second semble plus approprié que l'effacement du premier ${ }^{31}$ : autrement dit, (36) procède de (36a) et non de (36b). Il existe néanmoins des substantifs massifs pour lesquels aucun des deux prédéterminants n'est concevable seul:

(42) Luc a une de ces fièvres

(42a) * Luc a une fière

(42b) ${ }^{*}$ Luc a ces fièvres

Les contraintes inhérentes à la détermination standard ${ }^{32}$ de fièvre en tant que prédicat dans une construction à support (Luc a de la fièvre) expliquent les inacceptabilités ci-dessus.

La séquence déterminative un(e) de ces a la propriété remarquable d'imposer à certains noms avec lesquels elle se combine la forme du pluriel alors que généralement ils l'excluent en position prédicative; sur le plan sémantique, il ne s'agit pas pour autant d'une indication de pluralité du fait de la présence de un(e) en position initiale dans l'association de déterminants. Il s'ensuit que toutes sortes de prédicats nominaux 
sont compatibles avec l'association figée de déterminants, mais elle semble néanmoins totalement exclue avec certains:

(43) * Luc a éprouvé un de ces amours pour Léa

$(44){ }^{\star}$ Luc a ressenti une de ces joies ${ }^{33}$

De telles incompatibilités sont difficilement explicables: on constate, par défaut, qu'elles ne sont imputables ni à la valeur de la détermination figée (cf. infra), puisque d'autres actualisateurs des prédicats nominaux amour et joie l'autorisent (e.g. Luc éprouve un amour profond pour Léa, Luc ressent une joie intense), ni au fonctionnement de un de ces, étant donné qu'il opère sur des substantifs proches sur le plan syntactico-sémantique (Luc a eu une de ces peurs). Une explication plausible serait la différence de niveau de langue entre les deux types actualisateurs: les verbes supports éprouver et ressentir étant d'un niveau de langue plus soutenu que la détermination un de ces qui relève d'un registre familier. L'analyse n'est cependant que partiellement satisfaisante car la substitution des deux verbes par avoir autorise un(e) de ces uniquement dans le deuxième cas:

(43a) *? Luc a eu un de ces amours pour Léa

(44a) Luc a eu une de ces joies

Il faut donc aussi tenir compte de la nature et des particularités du prédicat nominal pour justifier sa combinatoire avec la séquence déterminative.

Du point de vue de sa structure de surface, la suite une de ces est ambiguë puisqu'elle peut aussi correspondre à une association libre de prédéterminants:

(36c) [Il y plusieurs sortes de migraines] Luc a attrapé une de ces migraines

Dans un tel cas, la nature des constituants peut varier:

(36d) [Il y plusieurs sortes de migraines] Luc a attrapé (une (de ces + des) migraines

(36e) [Il y plusieurs sortes de migraines] Luc a attrapé ((une + deux) de ces) migraines

Par contre, dans le cas où l'on a affaire à une association figée, le premier constituant est obligatoirement l'article indéfini singulier et le second le démonstratif pluriel.

Il a déjà été observé que celle-ci est souvent rattachée à des phrases exclamatives et que sa valeur relève de l'intensité forte (cf. Milner 1978, Tamba-Mecz 1981). Une autre signification grammaticale possible est le mélioratif (cf. infra):

(45) Luc a mangé un de ces gâteaux

Les oppositions détermination prédicative/détermination argumentale et substantif prédicatif/nom élémentaire contribuent à expliquer ses deux valeurs:

- l'intensif rattaché à un de ces s'observe quand il s'agit d'une détermination prédicative, i.e. au sein de constructions à support comme (36) et (42), mais peut concerner également la détermination argumentale pour peu que l'on ait affaire à un nom prédicatif:

(46) Si je buvais de cette piquette, Luc m'a prédit une de ces migraines

- le mélioratif en relation avec un de ces a trait à l'une ou l'autre détermination et, dans le cas de la détermination argumentale, il peut concerner aussi bien des noms élémentaires, e.g. (45), que des noms prédicatifs comme voyage: 
(47) Luc nous a proposé un de ces voyages

Le fonctionnement de l'association figée a été interprété par Tamba-Mecz (1981), d'une part, comme proche de celui d'une tournure partitive ou pseudo-partitive ${ }^{34}$ et, d'autre part, comme une réduction de une de ces espèces de $\mathrm{N}$ à un $(e)$ de ces $\mathrm{N}$ à laquelle est adjointe la notion de notoriété. Pour ce qui est du premier point, le rapprochement avec un GN donnant lieu à une interprétation partitive est problématique dans la mesure où, en cas de figement de la séquence déterminative, aucune des propriétés caractéristiques de la tournure partitive n'est observable; e.g. la dislocation droite de $\mathbf{C E ~} \mathbf{N}$ et la reprise conjointe par en:

(36f) * Ces migraines, Luc en a une ou la pronominalisation du GN démonstratif:

$(36 \mathrm{~g}) *[$ Ces migraines $]$ Luc a une de celles-ci

De plus, la possibilité d'une lecture partitive de $\boldsymbol{u n}(\boldsymbol{e})$ de ces $\mathrm{N}$ est sous-tendue par une lecture forte de l'article indéfini ainsi qu'une identification claire de ce que désigne ces $\mathbf{N}$. Pour ce qui est de $\boldsymbol{u n}(\boldsymbol{e})$, la lecture faible du prédéterminant est la plus vraisemblable quand il s'agit d'une association figée, comme l'attestent diverses propriétés qui contribuent à différencier les forts des faibles (Kleiber 2001):

(36h) ? Une de ces migraines est étudiée

(36i) ? Luc n'a pas une de ces migraines

En ce qui concerne ces, les occurrences discursives du seul GN démonstratif permettent, dans certains contextes, d'isoler dans un ensemble soit d'entités, soit d'occurrences d'une propriété dénotées par $\mathbf{N}$ un sous-ensemble de celles-ci indépendamment de tout fonctionnement déictique ou diaphorique ${ }^{35}$ :

(48) Max souffre d'une migraine. Luc connaît bien ces migraines

il n'est vraisemblablement pas question ici de ce type de fonctionnement du démonstratif car, comme l'indique le deuxième point, le GN démonstratif implique la notoriété dans la séquence déterminative un(e) de ces. Le déterminant démonstratif est donc ici un simple marqueur du caractère prototypique de la dénotation du substantif à l'instar d'autres types de déterminants, e.g. des modifieurs liés du type de $\mathbf{N}$ (cf. Buvet et Gross 1995):

(42c) Luc a une fièvre de cheval

La synonymie entre (42c) et (42) est fondée sur le fait que fièvre de cheval et ces fièvres font mention au parangon de la dénotation de fièvre: dans le cas de la suite $\mathbf{N} \boldsymbol{d e} \mathbf{N}$, une comparaison sous-tend cette correspondance; dans le cas de la suite ces $\mathbf{N}$, la mention est implicite puisqu'elle fait appel à un procédé qui n'est pas lexical mais grammatical.

Tamba-Mecz (1981) rappelle que les noms dont la forme pluriel est phonétiquement différenciée (un mal/des maux) restent à la forme singulier lorsqu'ils sont précédés de la détermination figée $u n(e)$ de ces (Luc a un de ces (mal + ${ }^{*}$ maux) de tête). On peut tirer deux conclusions sémantiques de cette observation, la seconde procédant en partie de la première: (i) ces est sans rapport avec une indication de pluralité et, étant donné son caractère unique, ces en se combinant avec $\mathbf{N}$ indique que l'on a affaire au parangon de la dénotation du substantif; (ii) au sein de la 
séquence déterminative figée, le déterminant démonstratif a donc un fonctionnement atypique et propre à cette séquence, il s'agit d'un marqueur de l'intensité forte ou du mélioratif, de telle sorte qu'en étant associé avec ces, l'article indéfini s'interprète comme un déterminant faible, i.e. il indique, par sa position frontale dans le GN, qu'il s'agit d'une occurrence non spécifiée de la dénotation de $\mathbf{N}$ lorsqu'elle est particulièrement remarquable soit en termes intensifs, soit en termes mélioratifs.

Le principe d'économie qui caractérise le langage explique vraisemblablement le fait que l'association figée un (e) de ces et la tournure partitive ont la même configuration et justifie la possibilité pour la première de fonctionner différemment de la seconde.

Des différentes descriptions et analyses des associations figées il ressort les points suivants :

- si les associations figées de déterminants donnent lieu aussi bien à une détermination prédicative qu'à une détermination argumentale, la répartition des séquences déterminatives se fait principalement au bénéfice de la première catégorie de détermination;

- les associations figées les plus fréquentes sont celles entre un prédéterminant donné et un modifieur obligatoire;

- les autres associations figées ont des configurations relativement proches mais s'avèrent avoir des fonctionnements très différents; trois types ont été différenciés: les premier et deuxième ressortissent respectivement à l'expression de la quantité et de l'intensité forte; le troisième se confond avec la seule séquence déterminative qu'il caractérise et qui correspond à marquer soit l'intensité forte, soit le mélioratif selon la nature des noms qui admettent cette détermination.

Avant de revenir sur la signification grammaticale de la détermination, nous traitons la question du figement des déterminants composés.

\subsubsection{Les prédéterminants composés figés}

Gross (1996) précise que «les notions de composition et de figement ne sont pas synonymes»: la composition a trait à des configurations d'éléments suffisamment stables pour que des valeurs constantes leur soient globalement associées; le figement résulte de l'impossibilité, totale ou partielle, de rendre compte des relations entre les différents constituants d'une structure donnée sur le plan syntaxique ou sémantique.

En ce qui concerne la détermination, il existe deux grands types de composés: les déterminants adverbiaux et les déterminants nominaux, i.e. des constructions formées respectivement d'un adverbe et d'un substantif suivis de la préposition de, la possibilité d'une détermination interne caractérisant également les noms déterminatifs $^{36}$ :

(49) Luc a beaucoup de livres (déterminant adverbial)

(50) Luc a une dizaine de livres (déterminant nominal)

Dans les deux cas, il s'agit de prédéterminants, la liste des adverbiaux comprend une cinquantaine d'éléments et plus 3000 noms déterminatifs ont été recensés (Buvet 1993). Les quelques autres composés sont des constructions atypiques comme je ne sais quel qui correspondent également à des prédéterminants.

Les prédéterminants composés qui ne sont pas figés ont un fonctionnement proche des prédéterminants simples: 
- ils sont le plus souvent distributionnellement peu restreints:

(49a) Luc a beaucoup de (livres + amis + bouteilles + chiens + énergie + maisons + sable $+\ldots)^{37}$

(50a) Luc a une dizaine de (livres + amis + bouteilles + chiens + *énergie + maisons + ${ }^{*}$ sable $+\ldots$ )

Les seules limitations qui affectent leur combinatoire sont en partie imputables aux mêmes types de contraintes de sélection qui caractérisent les prédéterminants simples; e.g. une dizaine de, à l'instar de plusieurs, est incompatible avec les noms dits massifs.

-en fonction des substantifs avec lesquels ils se combinent et de l'environnement phrastique de ces substantifs, ils appartiennent généralement à la catégorie des nondéfinis et s'interprètent comme des quantifieurs, des intensifs ou bien des aspectuels (cf. Buvet et Lim 1996):

(51) Luc achète beaucoup de chaussures (quantifieur)

(52) Luc éprouve beaucoup de honte (intensif)

(53) Luc reçoit beaucoup de gifles de la part de Léa (aspectuel: fréquentatif)

Les relations entre les différents prédéterminants composés non figés et les noms qu'ils précèdent sont analysables du fait que les constituants déterminatifs des types adverbial et nominal conservent les valeurs qui les caractérisent. Ainsi, lorsque l'on a affaire à un constituant nominal, il est possible «d'établir une relation syntaxique entre les deux noms et cette relation a une signification compositionnelle» (Gross 1996). Dans le cas de (50), diverses constructions, fondées sur des prédicats spécifiques, rendent compte du caractère non figé de la séquence déterminative une dizaine de:

Le nombre de livres que Luc a est une dizaine

Le compte des livres que Luc a s'élève à une dizaine

La possibilité de décrire dans les mêmes termes la relation entre des noms déterminatifs et les têtes nominales, dont ils dépendent syntaxiquement, a donné lieu à la constitution de 14 classes syntactico-sémantiques dans lesquelles sont repartis la plupart des déterminants nominaux (Buvet 1993; 1994; 2003a).

Les prédéterminants composés figés sont exclusivement des déterminants nominaux. Le figement qui les caractérise ressortit principalement à l'inanalysabilité de la relation entre le nom déterminatif et la tête nominale au sein du GN. Pour l'établir, on peut comparer deux déterminants nominaux formellement identiques dont l'interprétation varie cependant en fonction de la nature des substantifs avec lesquels ils se combinent:

(54) Luc a un tas de feuilles

(55) Luc a un tas d'ennuis

La classification des noms déterminatifs procède donc de la factorisation des prédicats qui caractérisent la relation entre les deux constituants principaux d'une suite DETa Na de Nb telle que DETa Na de est la détermination de Nb (Buvet 1993; 1994; 2003a). Ainsi, parmi les prédicats classifieurs des noms déterminatifs, il y a le couple former et constituer qui rend compte de la relation entre le $\mathbf{N a}$ tas et le $\mathbf{N b}$ feuilles dans (54): 
Les feuilles que Luc a forment un tas

Les feuilles que Luc a constituent un tas

Il s'ensuit que le substantif tas est sous-catégorisé dans la classe syntactico-sémantique des noms déterminatifs de 'forme' où l'on trouve également d'autres substantifs occupant la première place dans une suite DETa $\mathrm{Na}$ de $\mathrm{Nb}$ comme copeau, dé ou rondelle et tels que la relation entre $\mathbf{N a}$ et $\mathbf{N b}$ est aussi caractérisée par les prédicats classifieurs former et constituer. Dans (55), par contre, on ne peut pas décrire dans les mêmes termes la relation entre le nom déterminatif et la tête nominale:

* Les ennuis que Luc a forment un tas

* Les ennuis que Luc a constituent un tas

On a donc affaire à deux emplois du nom déterminatif tas: sur le plan sémantique, l'un est transparent, l'autre est opaque. C’est le second qui donne lieu à une séquence déterminative figée. Remarquons que le prédéterminant composé figé a un spectre distributionnel plus étendu que le prédéterminant composé non figé. Pour ce dernier, le nom déterminatif, tas dans (54), conserve la combinatoire qui le caractérise d'une façon générale, i.e. indépendamment de la construction déterminative DET Na de, et de ce fait, a des restrictions de sélection plus contraignantes. Pour autant, tous les noms déterminatifs qui ont au moins deux emplois ne donnent pas lieu à des séquences figées à large spectre distributionnel:

(56) Luc a acheté tout un train de (marchandises + fournitures + de produits de première nécessité + voitures + sable + bétails +)

(57) Luc a pris un train de (mesures + décisions + de réformes + ordonnances)

Le premier emploi de train, dont fait état (56), relève de la classe syntactico-sémantique des noms déterminatifs de 'contenant' (Buvet 1993 et Buvet 1995). À ce titre, il est constitutif d'un prédéterminant composé non figé qui, compte tenu des particularités de ce substantif, se combine avec des substantifs variés. Le deuxième emploi de train, illustré par (57), donne lieu à un prédéterminant composé figé qui est accepté, en tant que tel, par des substantifs sémantiquement différents en nombre beaucoup plus restreint.

Les prédéterminants composés figés se répartissent dans deux classes distinctes de déterminants nominaux, l'une et l'autre procédant de métaphores. La première est formée à partir de noms déterminatifs qui ont un autre emploi en tant que tel, la seconde de noms déterminatifs qui résultent de substantifs dont les autres emplois ne correspondent jamais à des déterminants (cf. Buvet 1993).

La première classe regroupe donc des déterminants nominaux figés dont les constituant principaux sont des substantifs comme tas dans (55), train dans (57) ou tonne dans le deuxième exemple ci-dessous:

(58) Luc a acheté une tonne de fonte

(59) Luc a une tonne de travail en retard

Le nom déterminatif tonne a deux emplois: le premier donne lieu à un prédéterminant composé non figé de la classe syntactico-sémantique dite des 'unités de mesure de masse' définie par le prédicat peser/poids:

La fonte que Luc a achetée pèse une tonne

Le poids de la fonte que Luc a achetée est d'une tonne 
La relation du second avec une tête nominale étant inanalysable dans les mêmes termes implique qu'il est constitutif d'un prédéterminant composé non figé.

* Le travail en retard de Luc pèse une tonne

* Le travail en retard de Luc est d'une tonne ${ }^{38}$

La deuxième classe est constituée de déterminants nominaux figés formés de substantifs qui n'ont qu'un seul emploi en tant que noms déterminatifs mais sont aussi d'origine métaphorique, i.e. ils ont au moins un autre emploi. Les sources des métaphores sont de nature diverse:

- agricole:

(60) La France a ramené une moisson de récompenses du festival de Venise - géologique:

(61) Luc a fait une montagne de fautes

- météorologique:

(62) Luc a versé un nuage de lait dans son thé - militaire:

(63) Luc a posé une rafale de questions à Max - religieuse:

(64) Une litanie de plaintes attendaient Luc

Selon Blanco (1998), plusieurs indices formels, dont l'application n'est cependant pas systématique, contribuent à la reconnaissance du figement de ces séquences déterminatives. Ainsi, la possibilité pour les noms déterminatifs de comporter des modifieurs internes comme authentique serait spécifique aux seuls déterminants nominaux métaphoriques du deuxième type:

(65) Une (E + authentique) marée de réfugiés attendaient la fin de la guerre

(65a) Une (E+ ${ }^{*}$ authentique) foule de réfugiés attendaient la fin de la guerre

Il en est de même pour la possibilité qu'ont les têtes nominales de dépendre de verbes qui comportent dans leur domaine d'arguments les substantifs à partir desquels ont été formées ces séquences déterminatives figées:

(66) Le chanteur a été submergé par un océan de scandales

(66a) * Le chanteur a été submergé par une centaine de scandales

Dans les cas où les têtes nominales sont prédicatives, de tels verbes peuvent également s'interpréter comme des verbes supports d'occurrence ou des opérateurs causatifs:

Un ouragan de passions parcourut le pays

La déclaration déclencha une avalanche de critiques

Pour terminer, précisons que des déterminants nominaux comme une kyrielle de ou une flopée de ne sont pas considérés comme des prédéterminants composés figés car, d'un point de vue synchronique, leur substantif n'a pas d'autre emploi que celui qui lui est rattaché dans la construction déterminative; leur interprétation est donc transparente et, par ailleurs, il n'est pas nécessaire d'expliquer leur fonctionnement en tenant compte de la relation entre le nom déterminatif et la tête nominale, ce qui les distingue des autres prédéterminants composés non figés. 


\section{La traduction des séquences déterminatives figées}

Dans un premier temps, nous évoquons la notion de signification grammaticale; dans un deuxième temps, nous présentons les différentes valeurs conventionnelles associées aux déterminants figés; dans un troisième temps, nous faisons état des représentations métalinguistiques que l'on peut associer aux phrases comportant une détermination totalement ou en partie figée. Dans un quatrième temps, nous montrons quelles en sont les conséquences pour la traduction automatique.

\subsection{La notion de signification grammaticale}

Pour illustrer cette notion, considérons la phrase suivante:

(67) Cet homme a donné une volée de gifles à un enfant

Ses différents constituants sont: le nom gifle que l'on interprète comme un prédicat; homme et enfant qui correspondent aux arguments du prédicat; a donné et une volée de qui constituent l'actualisation du prédicat; cet et un qui ont trait respectivement à l'actualisation des premier et deuxième arguments. Si le prédicat et ses arguments sont obligatoirement dotés d'une signification, dite lexicale, les différents actualisateurs sont quant à eux pourvus d'une signification, dite grammaticale. La première signification associe une seule valeur à un mot lexical en fonction de sa combinatoire, la seconde attribue une même valeur à toutes sortes de mots grammaticaux ou de morphèmes, lexicaux ou grammaticaux, compte tenu de leur environnement phrastique.

Les linguistes ont des points de vue différents sur la notion de signification grammaticale. De plus, bon nombre de catégories comme l'aspect, le mode ou la quantification ne sont pas décrites de façon unifiée. Les valeurs qui ressortissent à cette signification sont conventionnelles et en nombre fini. Si certaines sont universelles, d'autres sont propres à certaines langues ou familles de langue (cf. Melčuk 1993). Par ailleurs, pour une langue donnée, en l'occurrence le français, différents types d'actualisateurs peuvent partager une même valeur. Ainsi, dans (67), si on se limite à l'actualisation du prédicat, on observe, en termes de signification grammaticale, que le verbe support est non marqué, excepté du point de vue temporel, alors que la détermination s'avère porteuse de marques de l'intensité forte, de l'itératif et de la pluralité, cette dernière étant également spécifiée à l'écrit par la flexion du nom. Par contre, dans cette autre phrase, sémantiquement équivalente à (67), les deux premières valeurs sont imputables uniquement au verbe support (cf. Blanco et Buvet, à paraître) :

(67a) Un homme a assommé de gifles un enfant

Précisons enfin que les valeurs des différentes formes qui ressortissent à la signification grammaticale peuvent varier en fonction de la nature des éléments lexicaux faisant l'objet d'une actualisation. Par exemple, le déterminant nominal un tas de s'interprète uniquement comme un marqueur de la grande quantité plurielle devant voitures dans (68) et comme un marqueur à la fois de la grande quantité plurielle et de l'aspect itératif devant baisers dans (69):

(68) Luc possède un tas de voitures

(69) Luc envoie un tas de baisers à Léa 
Une partie des actualisateurs qui sont marqués en termes de signification grammaticale ont par ailleurs un caractère idiomatique qui se manifeste soit sous la forme de collocation, soit sous la forme d'expressions figées. Parmi ces dernières, figurent les modifieurs figés et les différentes séquences figées constituant une détermination.

\subsection{Signification grammaticale des déterminants figés}

Qu'il s'agisse de composants de détermination à caractère figé ou de détermination figée à part entière, les valeurs conventionnelles qui leur sont rattachées sont en partie les mêmes. Nous présentons, sous forme de tableau, chacune de ces valeurs, illustrée, s'il y a lieu, par une occurrence des trois types de déterminants figés étudiés ci-dessus.

\begin{tabular}{|c|c|c|c|}
\hline $\begin{array}{l}\text { Signification } \\
\text { grammaticale }\end{array}$ & $\begin{array}{l}\text { Modifieur figé } \\
\text { (exemple) }\end{array}$ & $\begin{array}{l}\text { Association } \\
\text { figée } \\
\text { (exemple) }\end{array}$ & $\begin{array}{l}\text { Prédéterminant composé } \\
\text { (exemple) }\end{array}$ \\
\hline intensité forte & $\begin{array}{l}\text { Des pluies torrentielles se } \\
\text { sont abattues sur la France }\end{array}$ & $\begin{array}{l}\text { Luc a eu une de } \\
\text { ces chances }\end{array}$ & $\begin{array}{l}\text { Luc a déversé un torrent } \\
\text { d'injures sur Max }\end{array}$ \\
\hline intensité faible & $\begin{array}{l}\text { Luc parle de dégustation } \\
\text { du bout des dents }\end{array}$ & $\begin{array}{l}\text { Luc a une } \\
\text { cervelle de } \\
\text { moineau }\end{array}$ & \\
\hline $\begin{array}{l}\text { grande } \\
\text { quantité }\end{array}$ & $\begin{array}{l}\text { Le patronat fait des } \\
\text { licenciements à jet continu }\end{array}$ & $\begin{array}{l}\text { Luc a du travail } \\
\text { à la pelle }\end{array}$ & $\begin{array}{l}\text { Léa a acheté des flots de } \\
\text { mousseline }\end{array}$ \\
\hline quantité faible & & & $\begin{array}{l}\text { Une poignée de gens sont } \\
\text { venus }\end{array}$ \\
\hline $\begin{array}{l}\text { aspect: } \\
\text { fréquentatif }\end{array}$ & & & $\begin{array}{l}\text { Luc m'a posé une foule de } \\
\text { questions }\end{array}$ \\
\hline $\begin{array}{l}\text { aspect: } \\
\text { duratif }\end{array}$ & $\begin{array}{l}\text { Luc est un ami de longue } \\
\text { date }\end{array}$ & & \\
\hline temporalité & & $\begin{array}{l}\text { Luc a fait une } \\
\text { arrivée ventre à } \\
\text { terre }\end{array}$ & \\
\hline mélioratif & Luc est un patron de choc & $\begin{array}{l}\text { Léa est une } \\
\text { fermme de coeur }\end{array}$ & \\
\hline péjoratif & $\begin{array}{l}\text { Luc était d'une humeur } \\
\text { massacrante }\end{array}$ & & \\
\hline
\end{tabular}

\subsection{Représentation métalinguistique et déterminants figés}

À diverses occasions (Buvet et Blanco 1999 et 2000; Buvet et Gavriilidou 2000), nous avons discuté dans la perspective de la mise en œuvre d'un système de traduction de la nécessité de proposer, via des dictionnaires électroniques monolingues coordonnés, des équivalents de traduction uniquement pour les prédicats et leurs arguments respectifs et de coder les actualisateurs sous une forme qui ne tient compte que de leur signification de telle sorte que la mise en correspondance automatique d'une phrase en langue source avec une phrase en langue cible ne soit pas affectée par le caractère fortement idiomatique de la plupart des actualisateurs.

Les différentes valeurs rattachées aux actualisateurs se présentent sous la forme soit d'indice (notamment quand il s'agit d'informations temporelles comme passé, futur, présent...), soit de prédicat métalinguistique (e.g. quand les valeurs sont l'intensité forte ou faible). Le dernier point présente l'avantage de proposer des équivalents 
de traduction qui ne sont pas dépendants des formes de départ de la langue source et de rattacher toutes sortes de constructions donnant lieu à des phrases synonymes à une même représentation métalinguistique. Ainsi, la phrase (67) admet comme synonyme non seulement (67a) mais aussi:

(67b) Cet homme a giflé de nombreuses fois et violemment un enfant

Dans ce dernier exemple, la forme du prédicat n'est plus un nom mais un verbe et le marqueur de l'aspect fréquentatif est un adverbe. Si l'on fait abstraction des indices relatifs au temps et à la détermination des arguments, les trois phrases sont donc associées à la représentation métalinguistique (RM) suivante:

RM65 = INT + \& ASPfré (gifl- (homme, enfant) $)^{39}$

Pour illustrer l'intérêt de ce type de représentation pour la traduction automatique, nous présentons plusieurs exemples tirés de l'étude comparée français-anglais de la détermination.

\subsection{Traduction franco-anglaise}

Considérons les exemples suivants:

(70) Luc a une fièvre de cheval

(71) Luc a une tonne de travail

(72) Luc avait une peur bleue

(73) Le parlement a voté un wagon de mesures

(74) Luc a acheté des livres à la pelle

La traduction automatique de ces phrases du français vers l'anglais n'est pas immédiate, notamment du fait que la détermination a un caractère idiomatique, qu'elle soit prédicative comme dans (70) à (72) ou bien argumentale comme dans (73) et (74). En termes de transposition, deux stratégies sont possibles: d'une part, donner en langue cible l'équivalent de traduction le plus proche possible de la langue source, d'autre part, proposer un équivalent de traduction approximatif mais correct, i .e. sans perte de sens significative. Dans l'un et l'autre cas, les techniques élaborées pour la traduction sont identiques, cf. Buvet et Blanco 2000, si ce n'est que le premier cas implique des ressources linguistiques très importantes. Il est question ici uniquement du second cas mais les équivalents de traduction que nous proposons constituent, à moindre coût, une avancée considérable par rapport aux transpositions des systèmes existants qui sont souvent de qualité moyenne ${ }^{40}$.

Le système auquel nous nous référons est un système modulaire constitué principalement d'un module d'analyse, d'un module de transfert et d'un module de génération $^{41}$; il met en rapport des langues relativement proches, e.g. deux langues romanes ou bien une langue romane et une langue germanique. Les rôles successifs des différents modules sont: (i) partir d'une phrase en langue source pour aboutir à sa représentation métalinguistique; (ii) associer la représentation métalinguistique de la langue de départ à une autre de la langue d'arrivée; (iii) transformer la nouvelle représentation métalinguistique en une phrase grammaticalement acceptable en langue cible.

Pour ce qui est de la première étape, il s'agit tout d'abord d'identifier puis d'étiqueter les prédicats et leurs arguments ainsi que les actualisateurs qui sont spécifiques aux uns et aux autres, e.g. la phrase (70) est analysée comme suit: 
(70a) $\{$ N0:= Npropre: Luc $\}$ \{actualisation prédicative: = Vsupport standard: avoir $\}$ \{actualisation prédicative: = DETintensif+: une _de cheval $\}$ PRED:= N: fièvr\}

La représentation est simplifiée car les informations de temps et de nombre ont été omises.

La première étape consiste également à faire état de la relation entre les arguments et les prédicats sous une forme fonctionnelle telle que les noms propres figurent sans aucune modification, les noms élémentaires en tant que lemmes et les prédicats en tant que racines. Les actualisateurs sont aussi pris en charge mais ils apparaissent sous une forme codifiée: lorsqu'il s'agit d'actualisateurs non standards, leur signification grammaticale non neutre est spécifiée sous forme d'indice relativement au prédicat ou à l'argument auquel ils sont rattachés; quand il s'agit d'actualisateurs, ils donnent lieu à une représentation fonctionnelle supplémentaire telle que la variable est la représentation fonctionnelle relative au prédicat et à ses éventuels arguments. Ainsi, les phrases étudiées sont associées aux représentations métalinguistiques suivantes:

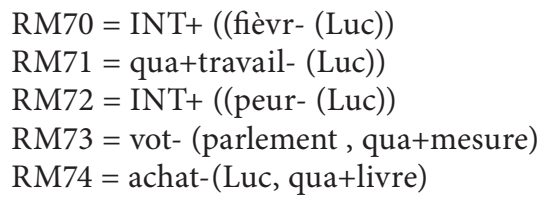

Le système reconnaît les prédicats et les arguments, il les interprète ensuite respectivement comme des fonctions et des variables; il identifie également:

-avoir dans (70) à (72) comme un verbe support standard à signification grammaticale nulle;

-une_de cheval dans (70), une tonne de dans (71) et une_bleue dans (72) comme trois occurrences de détermination prédicative telles que la signification grammaticale de la première et la troisième est l'intensité forte, celle de la deuxième la quantité importante;

-un wagon de dans (73), des_à la pelle dans (74) comme deux occurrences de détermination argumentale dont la signification grammaticale est la quantité importante ${ }^{42}$.

Les valeurs intensives des séquences déterminatives de (70) et (72) sont assimilées à un prédicat de niveau supérieur représenté sous forme de fonction dont les variables sont les représentations fonctionnelles relatives aux prédicats et aux arguments. La valeur quantitative de la détermination de travail dans (72) est représentée sous forme d'indice à l'instar de celle de la détermination de mesure dans (73) ou de livre dans (74). Quant aux actualisateurs à valeur nulle, ils n’apparaissent pas dans les représentations métalinguistiques.

La deuxième étape fait appel à des dictionnaires électroniques qui proposent un équivalent de traduction pour les arguments et les prédicats:

$$
\begin{aligned}
& \mathrm{RM}_{70}=\mathrm{INT}+((\text { fièvr- }(\mathrm{Luc})) \\
& \mathrm{RM}_{71}={ }_{\text {qua }}+\text { travail- (Luc)) } \\
& \mathrm{RM}_{72}=\mathrm{INT}+((\text { peur}-(\text { Luc })) \\
& \rightarrow \mathrm{RM}_{68^{\prime}}=\mathrm{INT}+((\text { fever- }(\mathrm{Luc})) \\
& \rightarrow \mathrm{RM}_{69},{ }_{\text {qua }}+\text { work- (Luc) } \\
& \rightarrow \mathrm{RM}_{70^{\prime}}=\mathrm{INT}+((\text { afraid }(\mathrm{Luc})) \\
& \left.\left.\mathrm{RM}_{73}=\text { vot- (parlement, }{ }_{\text {qua }}+\text { mesure }\right) \rightarrow \mathrm{RM}_{70^{\circ}}=\text { vote- (parliament, }{ }_{\text {qua }}+\text { mesure }\right) \\
& \mathrm{RM}_{74}=\text { achat-(Luc, }{ }_{\text {qua }}+\text { livre) } \\
& \rightarrow \mathrm{RM}_{70^{\circ}}=\text { buy- (Luc, }{ }_{\text {qua }}+\text { book) }
\end{aligned}
$$


Les prédicats sont spécifiés en tant que racines de telle sorte qu'il est possible de faire abstraction des catégories grammaticales pour associer les prédicats des deux langues, e.g. dans (71) et (72); les transpositions des noms travail et peur en français sont respectivement le verbe work et l'adjectif afraid en anglais. Les codes des actualisateurs sont conservés tels quels.

La première tâche de la troisième étape est de linéariser les prédicats et les arguments en fonction des spécificités de la langue cible, la seconde étant de leur attribuer des actualisateurs qui correspondent aux codes des représentations métalinguistiques, l'absence de code étant une indication d'un actualisateur à valeur neutre si sa présence est obligatoire. Les traductions obtenues sont les suivantes:

(70') Luc has a strong fever

(71') Luc works a lot ${ }^{43}$

(72') Luc was very afraid

(73') The parliament voted loads of measures

(74') Luc bought plenty of books

Des traductions plus élaborées sont possibles, e. g. Luc has a raging fever pour (70), pour peu que les bases de connaissances linguistes soient suffisamment complètes, ce qui n'est pas encore le cas pour l'anglais en ce qui concerne la détermination à caractère idiomatique. Par contre, la description de la détermination figée du français est suffisament avancée pour être exploitée en traduction automatique, i.e. dans le système mentionné au niveau du module d'analyse.

\section{NOTES}

1. Une phrase élémentaire est constituée d'un seul prédicat sous-jacent si elle comporte au moins un prédicat supplémentaire. Une phrase non élémentaire comprend au moins deux prédicats dont aucun n'est sous-jacent à l'autre. Il existe différents niveaux prédicatifs selon la nature des schémas d'arguments, i.e. soit non phrastique ou partiellement phrastique, soit totalement phrastique. Dans les phrases élémentaires, les prédicats correspondent principalement à des verbes, des noms ou des adjectifs, occasionnellement à des prépositions, e.g. sous dans La chaise est sous la table.

2. Une conséquence de cette définition c'est que l'absence de détermination est assimilée à un prédéterminant dit article zéro (Anscombre 1986a et 1986b) et noté ZERO.

3. Cette distinction ne recoupe pas celle entre les modifieurs libres et les modifieurs figés (cf. infra).

4. Un troisième niveau est également concevable, le niveau infra-phrastique (J'ai rencontré un ami d'un voisin); (cf. Buvet 2002a et 2003a). Il n'en est généralement pas tenu compte car ce dernier niveau est rattaché à l'un des deux autres niveaux du fait qu'il concerne des déterminants constitutifs des modifieurs, ces derniers et les prédéterminants dont ils dépendent ressortissant au niveau soit interphrastique, soit intraphrastique. La prise en compte du niveau infra-phrastique est parfois nécessaire pour l'analyse des deux autres (cf. Corblin 1987; Flaux 1992 et Milner 1982).

5. Un nom élémentaire est un substantif qui ne fonctionne jamais comme un prédicat, un nom prédicatif fonctionne comme un prédicat dans une construction à support.

6. Remarquons que l'assimilation de l'absence de toute détermination à un prédéterminant simple, l'article zéro, a comme conséquence que la détermination d'un nom uniquement précédé ou suivi d'un modifieur est complexe (e.g. dans J'ai grand- faim, on considère que le déterminant est du type ZERO MODIF, i.e. l'article zéro suivi d'un modifieur). Par ailleurs, dans Luc fait sa promenade, le fait que le possessif a un fonctionnement différent ne relève pas du figement; il s'agit d'un emploi différent (cf. Buvet 2003b).

7. Les numéraux composés sont considérés comme des associations libres de déterminants simples (e.g. trente- trois est assimilé à un déterminant complexe résultant de l'association des déterminants simples trente et trois).

8. Des variations limitées sont possibles, elles portent généralement sur un seul constituant (e.g. Luc a acheté (un+ trois) kilos de pommes). 
9. En l'occurrence, celui de constituant de séquence déterminative.

10. Les exemples de cette section sont empruntés à la littérature du lexique-grammaire sur le sujet (Danlos 1981; Gross 1988 et 1990; Gross 1982; 1985 et 1988).

11. Selon Corblin (1987), la combinaison de l'article indéfini avec un substantif permet de faire mention à un nouveau référent sur la base d'une extraction.

12. La nature proverbiale de cette phrase explique vraisemblablement le fonctionnement régulier des déterminants (Anscombre 2003).

13. Les modifieurs sont de diverses natures en tant qu'extensions du nom. On distingue généralement les catégories suivantes: les adjectifs épithètes (un enfant mécontent), les noms épithètes (une tarte maison), les compléments du nom (un enfant de cette école), les relatives (un enfant qui dort), les participiales (un enfant réveillé) et les infinitives (un enfant à prendre au sérieux) (cf. Riegel et al. 1994).

14. Certains modifieurs composés sont également des noms épithètes comme beurre frais relativement à gant (Gavriilidou 1998).

15. Rappelons que les adjectifs épithètes sont soit simples (e.g. rouge dans Luc a une voiture rouge), soit complexes (Luc a une voiture bleue roy), ces derniers se subdivisant en adjectifs composés (Luc a une voiture d'une couleur indéfinissable) et adjectifs composés figés (Luc a une voiture de seconde main).

16. Le plus souvent, ils sont associés à un seul substantif ou bien quelques uns.

17. C'est une détermination du type ZERO MODIF.

18. Un autre prédéterminant est possible avec à sa mémère: le générique le.

19. Le possessif doit être coréférent au sujet du verbe dont dépend le GN constitué de ce déterminant (cf. Buvet 2002b).

20. Par contre, aussi bien le GN débutant par la plupart que celui commençant par le plus clair peuvent faire l'objet d'une mise en apposition en tête de phrase: La plupart des livres, Luc les a lu; Le plus clair de son temps, Luc le passe à dormir.

21. Il existe une série limitée de noms qui sont substituables au nom dans passer le plus clair de son temps: existence, vie, etc.

22. Le prédicat temps est ici un prédicat complexe tel que $\mathbf{N} 0$ correspond nécessairement à un GN relatif à un humain ou à un animal et $\mathbf{N} 1$ une construction infinitive dont le sujet effacé est coréférent au premier argument et le verbe relatif à une activité. Il ne s'agit pas d'une séquence figée étant donné que le seule nom prédicatif peut figurer dans un GN indépendamment des autres constituants de la construction à support: Luc passe le plus clair temps de son temps à dormir. Ce temps, il ferait mieux d'en profiter autrement.

23. On aura plus difficilement:?[en matière de bêtise] Le comble, c'est d'agir ainsi; [en matière de goujaterie] Le summum, c'est de se comporter de la sorte.

24. La notion de 'prélèvement quantitatif indéfini' (Dubois et al. 1994) est inappropriée pour les noms abstraits.

25. «Nous appellerons typants les prédicables qui déterminent la classe des individus particuliers qui sont leurs occurrences. Parallèlement, nous définirons leur usage prédicatif comme l'assignation d'un type» (Riegel 1985).

26. Éventuellement, on peut considérer qu'il s'agit d'une telle relation si l'ensemble est unaire.

27. La forme un devant comble est attestée dans: C'est un comble! mais, par contre, la suite un comble semble exclue en tant que premier constituant d'une association figée de déterminants: ${ }^{*}$ Agir ainsi est un comble de la bêtise.

28. Les noms comble et summum ont la particularité sémantique de marquer le 'haut degré' et la propriété syntaxique d'être obligatoirement saturés par un substantif de telle sorte que, relativement à la dénomination de ce dernier, ils indiquent une occurrence.

29. Pour ce qui est de comble, une autre signification grammaticale s'associe à celle de l'intensité forte: le péjoratif.

30. La séquence le comble de peut également être précédée de la préposition à dans une construction en être avec certains noms d'émotion: Luc est au comble de la joie.

31. Nonobstant la perte de sens due à cette reconstruction.

32. La détermination standard est une détermination neutre du point de vue de la signification grammaticale (cf. Buvet et Blanco, à paraître).

33. Si la phrase Luc ressent une de ces joies inespérées est grammaticale, c'est parce que la présence du modifieur adjectival interdit d'interpréter une de ces comme une détermination figée. 
34. Une construction est dite pseudo-partitive quand le substantif est étranger à toute notion de quantité.

35. Selon Riegel et al. (1994:156), ce fonctionnement du déterminant démonstratif implique 'une caractérisation évaluative'.

36. Les noms déterminatifs sont des substantifs pouvant donner lieu à des déterminants nominaux.

37. Certains adverbiaux ont une portée distributionnelle moindre que d'autres, e.g. infiniment de.

38. Un autre critère permet de distinguer les prédéterminants composés figés des non figés: les premiers ont une détermination interne qui prête à variation (Luc a acheté (une + des + plusieurs + trois) tonnes de fonte) mais pas les seconds, ou alors d'une façon très limitée (Luc a (une + des + plusieurs + trois) tonnes de travail en retard); cf. Buvet 2003a.

39. INT+ symbolise la valeur correspondant à l'intensité forte, ASPfré la valeur relative à l'aspect fréquentatif, \& étant un opérateur de concaténation.

40. Par exemple, le système Reverso traduit (71) par Luc is one ton of work, ce qui est erroné.

41. Il s'agit, pour l'instant, d'un prototype fondé sur la technologie INTEX (Silberztein 1993).

42. Le parlement étant reconnu comme une entité nommée, le n’est pas interprété comme la détermination de parlement, i.e. la séquence est indécomposable.

43. Luc has a lot of work est également une traduction possible.

\section{RÉFÉRENCES}

Anscombre, J.-C. (1986a): «Larticle zéro en français: un imparfait du substantif?», Langue française 72 , Paris, Larousse.

Anscombre, J.-C. (1986b): «Article zéro, termes de masse et représentation d'événements en français contemporain ", Recherches linguistiques 11, Paris, Klincksieck, pp. 5-34.

Anscombre, J.-C. (2003) : «Les proverbes sont-ils des expressions figées?», Cahiers de lexicologie 82, pp. 159-173.

Blanco, X. (1998): «Les déterminants nominaux figés -Perspective contrastive espagnolfrançais», Actes de la première Rencontre Linguistique Méditerranéenne, Tunis.

Blanco, X. et P.-.A. Buvet (1999): «À propos de la traduction automatique des déterminants de l'espagnol et du français», Meta 44-4, pp. 525-545.

Buvet, P.-A. (à paraître): Remarques sur la détermination du français.

Buvet, P.-A. et G. Gross (1995): «Comparaison et expression du haut degré dans le groupe nominal», Faits de langues 5, pp. 83-88.

Buvet, P.-A. et X. Blanco (2000): «De l'analyse syntactico-sémantique du lexique à la traduction automatique», BULAG 25, pp. 69-87.

Buvet, P.-A. et X. Blanco (à paraître): «Verbes supports et significations grammaticales en espagnol - Implication pour la traduction ", Lingvisticae Investigationes.

Buvet, P.-A. et Z. Gavriilidou (2000): "Comment traduire les bruits. "Projet DETTAL" ", in Mejri, S., Baccouche, T., Clas, A. et G. Gross (dir.), La traduction: théories et pratiques. Actes du Colloque international Traduction humaine, Traduction automatique, Interprétation, (Tunis, 28-29-30 septembre 2000), pp. 21-55.

Buvet, P.-A. (1993): Les déterminants nominaux quantifieurs, Thèse de Doctorat, Université Paris 13, Paris.

Buvet, P.-A. (1994): «Détermination: les noms», Lingvisticae Investigationes 18-1, pp. 121150.

Buvet, P.-A. (1998): «Détermination et classes d’objets», Langages 131, pp. 91-102.

Buvet, P.-A. (2002a): "Analyse de compléments du nom en termes de classes d'objets», Le Français Moderne 70-2, pp. 187-209.

Buvet, P.-A. (2002b) : «Le défini obligatoirement modifié», Langages 115, pp. 97-125.

Buvet, P.-A. (2003a): «La construction déterminative DET N de», Syntaxe et Sémantique 5, pp. 71-90.

Buvet, P.-A. (2001): «Les déterminants intensifs», in Blanco, X., Buvet, P.-A., GavriIlidou, Z., (dir.), Détermination et formalisation, Lingvisticae Investigationes Supplementa 23, pp. 101-113. 
Buvet, P.-A. et J.-H. Lim (1996) : «Les déterminants nominaux aspectuels», Lingvisticae Investigationes 20-2, pp. 271-285.

Buvet, P.-A. (2003b) : «La possessivation dans les constructions à support», Lingvisticae Investigationes 26-1, pp. 47-70.

Corblin, F. (1987): Défini, indéfini et démonstrastif, Genève-Paris, Droz.

Danlos, L. (1981): «La morphosyntaxe des expressions figées», Langages 63, pp. 53-74.

Dubors, J. et al. (1994): Dictionnaire de linguistique, Paris, Larousse.

Flaux, N. (1992): «Les syntagmes nominaux du type le fils d'un paysan: référence définie ou indéfinie», Le Français Moderne 60-1, pp. 23-45.

Gavrillidou, Z. (1998): Étude comparée - les suites NN en français et en grec, Lille, Septentrion.

Gross, G. (1988): «Degré de figement des noms composés», Langages 90, pp. 57-72.

Gross, G. (1990): «Définition des noms composés dans un lexique-grammaire», Langue française 87, pp. 84-90.

Gross, G. (1994): Classes d'objets et description des verbes, Langages 115, pp. 15-30.

Gross, G. (1995a): «Une sémantique nouvelle pour la traduction automatique: les classes d'objets", La tribune des industries de la langue et de l'information électronique 17-18-19.

Gross, G. (1995b): «Prédicats nominaux et compatibilité aspectuelle», Langages, 121.

Gross, G. et R. Vives (1986): «Les constructions nominales et l'élaboration d'un lexiquegrammaire», Langue française 69, pp. 5-27.

Gross, M. (1985): «Sur les déterminants dans les expressions figées», Langages 79, pp. 89-117.

Gross, M. (1982): «Une classification des phrases figées du français», Revue québécoise de linguistique 11-2, pp. 151-158.

Gross, M. (1988): «Les limites de la phrase figée», Langages 90, pp. 7-22.

Kleiber, G. (1986): "Adjectif démonstratif et article défini en anaphore fidèle», Recherches linguistiques 11, pp. 169-184.

Kleiber, G. et R. Martin (1977): «La quantification universelle en français», Semantikos 2-1, pp. 19-36

Kleiber, G. (2001)::«Déterminants indéfinis ou quand les faibles jouent aux forts », Lingvisticae Investigationes Supplementa 20, pp. 195-218.

Le Pesant, D. et M. Mathieu-Colas (1998): «Introduction aux classes d'objets», .Langages 131, pp. 6-33.

MejRI, S. (1997): Le figement lexical: descriptions linguistiques et structuration sémantique, Série linguistique X, Publications de la Faculté des lettres de la Manouba.

MeL'ČUK, I. (1993): Cours de Morphologie Générale (théorique et descriptive), vol 1 Introduction et première partie: le mot, Montréal/Paris, Les Presses de l'Université de Montréal/CNRS.

Milner, J.-C. (1978): De la syntaxe à l'interprétation, Paris, Seuil.

Milner, J.-C. (1982): Ordres et raisons de langue, Paris, Seuil.

RiegeL, M. (1985): L'adjectif attribut, Paris, PUF.

Riegel, M. (1988): «L'adjectif attribut de l'objet du verbe avoir -amalgame et prédication complexe», Travaux de linguistique 17, pp. 69-87.

RIEGeL, M. et al. (1994): Grammaire méthodique du français, Paris, PUF.

Silberztein, M. (1993): Dictionnaires électroniques et analyse automatique de textes. Le système INTEX, Paris, Masson.

Tамва-Mecz, I. (1981): «Un de ces... », L'information grammaticale 11, pp. 3-6. 